\title{
Análise do Programa Agentes Locais de Inovação no Processo de Inovação das Micro e Pequenas Empresas do Setor de Comércio Varejista do Estado de Pernambuco
}

\author{
Analysis of the Agentes Locais de Inovação Program in the Innovation \\ Process of Micro and Small Enterprises of the Pernambuco State Retail \\ Trade Sector
}

\author{
Patricia da Silva Nogueira ${ }^{1}$ \\ Sueli Menelau de Novais ${ }^{1}$ \\ Alessandra Sousa Cordeiro de Sá ${ }^{1}$ \\ Evelyn Siqueira Bezerra da Silva ${ }^{1}$ \\ ${ }^{1}$ Universidade Federal de Pernambuco, Recife, PE, Brasil
}

\begin{abstract}
Resumo
O Programa Agentes Locais de Inovação (ALI) objetiva ajudar as Micro e Pequenas Empresas (MPEs) na melhoria de ações voltadas para inovação, seguindo as indicações do Serviço Brasileiro de Apoio à Micro e Pequena Empresa (SEBRAE), que atua em acordo com a política pública de inovação voltada a promover o crescimento econômico das empresas atendidas. O objetivo do estudo foi analisar como o Programa ALI está favorecendo o avanço de ações de inovação de MPEs do setor de comércio varejista de vestuário, na Região Metropolitana de Recife, no ciclo 2015-2017. O estudo possui abordagem quantitativa e finalidade descritiva, e coleta estruturada e longitudinal. O questionário é o instrumento aplicado pelo Programa ALI e se fundamenta na ferramenta Radar de Inovação. Os resultados indicam que o objetivo do Programa ALI foi alcançado, pois as MPEs estão saindo da posição de desenvolvedoras de inovação, indo para a posição das que inovam sistematicamente.
\end{abstract}

Palavras-chave: Política Pública de Inovação. SEBRAE. Programa ALI.

\begin{abstract}
The Agentes Locais de Inovação Program (ALI) aims to help micro and small enterprises (MSEs) in the improvement of actions geared toward innovation, following the indications of the Serviço Brasileiro de Apoio à Micro e Pequena Empresa, which operates in accordance with the public policy of innovation to promote the economic growth of enterprises. The objective of this study was to analyze how the Program ALI is favoring the advancement of innovation actions of MSEs in the industry of retail trade of clothes, in the metropolitan region of Recife, in the 2015-2017 cycle. The study has a quantitative approach and descriptive purpose, and structured collection and longitudinal. The questionnaire is the instrument applied by the Program ALI and is based on the Innovation Radar tool. The results indicate that the goal of the Program ALI was achieved because the MSEs are leaving the position of innovation, developers headed to the position of that innovate systematically.
\end{abstract}

Keywords: Public Policy of Innovation. SEBRAE. ALI Program.

Área Tecnológica: Propriedade Intelectual. Inovação. Desenvolvimento 


\section{Introdução}

Diante do crescimento da competição com o processo de aberturas de mercados, o comércio eletrônico e o fenômeno da globalização, a inovação tem sido amplamente discutida nas empresas de setores diversos, sendo apontada como um inestimável ativo para aquelas que conseguem agregar valor a novos produtos ou serviços que se diferenciem dos oferecidos (CARVALHO, 2009; KON, 2015; PORTER, 1999). De acordo com Figueiredo (2013), a base da competitividade de um país está em seu papel criador de um ambiente favorável à inovação.

Com a crescente velocidade das mudanças, o conhecimento e a inovação tornam-se ferramentas para a competitividade das organizações, podendo ser o grande diferencial para se garantir a sobrevivência empresarial em qualquer setor (SCHUMPETER, 1988). Nesse contexto, Porter (1999, p. 167) destaca que "[...] a competitividade de um país depende da capacidade [...] de inovar e melhorar. As empresas conquistam uma posição de vantagem em relação aos melhores competidores do mundo em razão das pressões e dos desafios".

As economias de serviços, o chamado setor terciário, se expandem e se difundem, principalmente, por meio de atividades produtivas relacionadas ao comércio (KON, 2015). Nesse ambiente de negócios e desenvolvimento, emerge a importância do varejo no cenário econômico brasileiro, que vem sendo cada vez mais reconhecido e destacado em sua atuação local e regional nas economias nacionais (KON, 2015).O crescimento do setor trouxe especialmente a partir de 2017, um aumento no volume de empregos formais no País, apresentando indicadores de modernização das empresas por meio de novos serviços e o uso de algumas tecnologias, como, aplicativos. Sendo o setor mais representativo na economia brasileira correspondendo a $70 \%$ do Produto Interno Bruto (PIB) brasileiro, segundo dados do Instituto Brasileiro de Geografia e Estatística (IBGE, 2017). Assim, são considerados desse setor de serviços, por exemplo, transporte, comércio, limpeza, alimentação, hospedagem e beleza.

De acordo com as métricas emitidas, chama a atenção: o número de estabelecimentos de Micro e Pequenas Empresas (MPEs), no setor de comércio do Brasil (49,4\% do total); o índice de $41 \%$ de emprego da população brasileira desse setor, sendo 16,2 milhões de empregos formais - cerca de $52 \%$ do empregos ofertados no País da geração de postos de trabalho (SEBRAE; DIEESE, 2013).Os governos nacionais passam a observar que entre os motivos que ocasionam taxas de crescimento elevadas em alguns países está a preponderância da inovação ao longo da cadeia produtiva, e a sua difusão passa então a ser entendida como uma questão-chave (CARVALHO, 2009). E é consenso que no contexto de economias emergentes, como o Brasil, uma agenda pública indutora de inovação e a gestão da inovação nas empresas ainda precisam ser construídas (FIGUEIREDO, 2013; KON, 2015). No campo de políticas de inovação, o Programa Agentes Locais de Inovação (Programa ALI), do Serviço Brasileiro de Apoio às Micro e Pequenas Empresas (SEBRAE); em parceria com o Conselho Nacional de Desenvolvimento Científico e Tecnológico (CNPq), procura desde 2008 inserir a cultura da inovação no ambiente organizacional das MPEs, por meio tanto da transferência de tecnologia, quanto do desenvolvimento de projetos de pesquisa.

O objetivo do Programa ALI é estimular a inovação na cultura empresarial das MPEs, promovendo a ampliação da sua capacidade competitiva com a utilização da ferramenta Radar da Inovação (SEBRAE, 2010), adaptada à realidade brasileira. Essa ferramenta, segundo Bachmann e Destefani (2008), permite mostrar as oportunidades relacionadas ao tema da inovação que 
as empresas dispõem, no sentido de torná-las mais competitivas, melhorar a sua rentabilidade e aumentar a qualidade dos produtos ofertados, e, segundo o $\operatorname{SEBRAE}(2010$, p. 6), " [...] a metodologia adotada admite que a inovação não é um evento ou fato isolado, mas fruto de um processo. Daí a preocupação em avaliar não o simples resultado (número de inovações), mas a maturidade do processo de gestão da inovação das empresas".

O desafio do Programa ALI reside em desmitificar o conceito de inovação, e, ao mesmo tempo, sensibilizar e mobilizar os empresários quanto à importância de inovar em um mercado dinâmico e globalizado, mostrando novas perspectivas de negócios e o possível acesso às formas de oportunidades no âmbito da inovação (BES; KOTLER, 2011). Em Pernambuco (PE) as MPEs correspondem a 158.017 estabelecimentos, os quais representam $98,6 \%$ do total de empresas do estado. O setor mais representativo é o de comércio e serviços com $84,1 \%$, seguido dos setores de indústria $11,7 \%$ e construção $4,2 \%$, representando cerca de $26 \%$ no Produto Interno Bruto (PIB) de Pernambuco, de acordo com os dados do IBGE (2017), demonstrando que ainda há espaço para o crescimento dessas empresas no desempenho econômico do País.

Com relação à geração de emprego no estado, o comércio se destaca como o setor de atividade econômica mais geradora de empregos (SEBRAE; DIEESE, 2013) e, em uma análise do mercado local da Região Metropolitana do Recife, verificou-se que o setor é composto em sua maioria de MPEs, empresas que possuem de 0 a 9 pessoas ocupadas, e a receita bruta anual é menor ou igual a até 360 mil reais. Com base nesse contexto delimitou-se como objetivo deste texto analisar como o Programa ALI está favorecendo o avanço de ações de inovação nas MPEs. Especificamente, por meio da observação dos estágios propostos pelo Programa ALI, pretendeu-se investigar as empresas do setor de comércio e serviços de cidades que compõem a Região Metropolitana de Recife, que participaram do Programa ALI no último ciclo (2015-2017).

\section{Revisão da Literatura}

Áreas do conhecimento distintas, como Engenharias, Química, Economia e Administração estudam e restringem o conceito de inovação (CARVALHO, 2009). Existem várias definições para inovação, a mais difundida afirma que a inovação é a implementação de um produto (bem ou serviço) novo ou significativamente melhorado, ou um processo, ou um novo método de marketing, ou um novo método organizacional nas práticas de negócios, na organização do local de trabalho ou nas relações externas (OCDE, 2007). Se faz necessário estar intrínseco o conhecimento em relação ao entendimento durante as atuações ou avaliações de inovação, ter em consenso seu significado, para poder exercer na prática sua aplicabilidade nas empresas (CARVALHO, 2009).

Schumpeter (1988) observa também que quanto ao grau de novidade, são dois tipos de inovação: a chamada radical, ou seja, aquela que produz grande impacto econômico ou mercadológico; e a incremental, que alude aos aprimoramentos técnicos os aprimoramentos técnicos de base contínua. E embora a descontinuidade da inovação radical provoque grandes mudanças na sociedade, a incremental tem sua importância no processo inovativo (ANDREASSI, 2007).

Sobre a forma de se avaliar a inovação, existem diversas. O diagnóstico de inovação objetiva medir o grau de inovação em que a empresa se encontra e apontar quais atividades inovadoras estão sendo desenvolvidas pela empresa (CARVALHO et al., 2015; CAVALCANTI 
FILHO; OLIVEIRA; CAVALCANTI, 2012). Schumpeter (1988) propõe que para mensurar a inovação de uma organização sejam observadas as dimensões: introdução de novos produtos, melhores métodos de produção, novos mercados, desenvolvimento de novas fontes provedoras de insumos e criações de novas estruturas de mercado em uma indústria. Uma contribuição que foi popularizada e se tornou em uma das ferramentas mais utilizada para essa finalidade está baseada no instrumento Radar da Inovação, desenvolvido inicialmente com 12 dimensões, por Sawhney, Wolcott e Arroniz (2006), ampliado posteriormente com mais uma dimensão (ambiência inovadora) por Bachmann e Destefani (2008) (PAREDES; SANTANA; FELL, 2014). Como pode ser visto no Quadro 1 a seguir, o Radar da Inovação busca apontar 13 áreas que uma empresa pode procurar oportunidades para inovar.

Quadro 1 - Dimensão e definições do Radar da Inovação

\begin{tabular}{|c|c|c|}
\hline $\mathbf{N}^{\mathbf{O}}$ & Dimensão & DEFINIÇÃo \\
\hline 1 & Oferta & $\begin{array}{l}\text { Se refere ao desenvolvimento de produtos ou } \\
\text { serviços com características inovadoras. }\end{array}$ \\
\hline 2 & Plataforma & $\begin{array}{l}\text { Relaciona-se com a adaptabilidade do sistema de produção } \\
\text { face à diversidade de produtos demandados. }\end{array}$ \\
\hline 3 & Marca & É a forma de como as empresas transmite aos clientes a sua imagem. \\
\hline 4 & Clientes & Busca identificar necessidades dos clientes ou novos nichos de mercado. \\
\hline 5 & Soluções & $\begin{array}{l}\text { São os sistemas, ou mecanismos, para simplificar as } \\
\text { dificuldades do cliente e solucionar problemas. }\end{array}$ \\
\hline 6 & Relacionamento & Relaciona-se com a experiência do cliente ao interagir com a empresa. \\
\hline 7 & Agregação de valor & $\begin{array}{l}\text { Busca melhorar a forma de captar o valor dos } \\
\text { produtos percebido por cliente e fornecedores. }\end{array}$ \\
\hline 8 & Processos & $\begin{array}{l}\text { Vislumbra o redesenho dos processos produtivos, de modo } \\
\text { a permitir incremento de eficiência operacional. }\end{array}$ \\
\hline 9 & Organização & Visa melhorar a estrutura da empresa. \\
\hline 10 & Cadeia de fornecimento & $\begin{array}{l}\text { É a busca por incrementar a logística com os } \\
\text { fornecedores e clientes, seja interno ou externo. }\end{array}$ \\
\hline 11 & Presença & $\begin{array}{l}\text { Relaciona-se com a identificação de novas formas } \\
\text { de comercialização e ou distribuição. }\end{array}$ \\
\hline 12 & Rede & $\begin{array}{l}\text { É a comunicação entre os elos da cadeia de fornecimento, } \\
\text { que conecta a empresa e seus produtos, aos clientes. }\end{array}$ \\
\hline 13 & Ambiência inovadora & $\begin{array}{l}\text { Relaciona-se com os profissionais que compõem a } \\
\text { empresa e que colaboram com a cultura da inovação, } \\
\text { criando um ambiente propício à inovação. }\end{array}$ \\
\hline
\end{tabular}

Fonte: Elaborado pelos autores deste artigo, a partir de Bachmann e Destefani (2008)

Do mesmo modo não existe ainda consenso na literatura sobre o conceito ou a definição de políticas públicas, por este ser ainda um campo recente da ciência política. Em geral, entende-se políticas públicas como o instrumento ou conjunto de ação dos governos (SOUZA, 2006), uma ação elaborada no sentido de enfrentar um problema público (SECCHI, 2012) ou um "[...] conjunto de decisões e ações destinadas à resolução de problemas políticos." (RUA, 1998, p. 731). Então, pode-se definir que políticas públicas são conjuntos de programas, ações $e$ atividades desenvolvidas pelo Estado diretamente ou indiretamente, com a participação de 
entes públicos ou privados, que visam assegurar determinado direito de cidadania, de forma difusa ou para determinado seguimento social, cultural, étnico ou econômico (FARAH, 2013).

Além disso, é necessário analisar a atuação das políticas públicas de forma mais ampla, já que há uma participação cada vez mais intensa do setor privado no ecossistema, tanto como atores de financiamento e investimento, com apoio inicial à inovação, provendo novas formas de colaboração e troca de boas práticas com empresas, quanto como intermediadores em programas públicos, como é o caso do SEBRAE (OCDE, 2015). Isso inclui ações mais específicas para atividades, como o desenvolvimento de redes, o alinhamento de prioridades entre diferentes atores, a construção de novas capacidades institucionais e a promoção de sinergias entre as diferentes partes interessadas (WARWICK, 2013).

\section{Metodologia}

A abordagem da pesquisa é quantitativa e longitudinal, do tipo descritiva, e os dados primários coletados são advindos de uma coleta estruturada em nível de análise (HAIR et al., 2005). A população do estudo são empresas atendidas pelo Programa ALI de PE do comércio varejista. A metodologia de avaliação do Programa ALI prevê que a avaliação utilizando o Radar da Inovação das empresas atendidas seja realizada em três momentos temporais distintos, compondo um ciclo de atendimento, sendo denominados de R0, R1 e R2. A meta é analisar, assim que possível e mensurar o índice de desenvolvimento e crescimento evolutivo da inovação que a empresa participante conseguiu obter no mercado em que atua.

Desse modo a amostra inicial foi composta de 50 empresas, cerca de $20 \%$ das atendidas, foram as que completaram o ciclo do Programa ALI, sendo elas do setor de comércio varejista do vestuário, localizadas em seis bairros, sendo eles de Piedade - Jaboatão dos Guararapes, Pina e Boa Viagem - Zona Sul do Recife, Espinheiro e Aflitos - Zona Norte do Recife, Bairro Novo - Olinda, bairros de classe média da Região Metropolitana de Recife, Olinda e Jaboatão dos Guararapes (outras cidades importantes e com crescente desenvolvimento comercial). A amostra final estudada, entretanto, resultou de $20 \%$ do número inicial, sendo 10 empresas do comércio varejista do vestuário, pois foram apenas essas que cumpriram os três momentos temporais do ciclo de avaliação. Portanto, o critério de seleção amostral é não probabilístico e por conveniência (HAIR et al., 2005).

Para manter a confidencialidade das informações prestadas pelo Programa ALI, no momento inicial e final do processo, as empresas foram denominadas de E1, E2 ... E10. O questionário empregado foi aplicado pelos agentes do Programa ALI, e foi estruturado em 42 questões fechadas, que abarcam as 13 dimensões presentes no Quadro1, com o intuito de compreender o ambiente interno e externo das organizações, quanto ao desenvolvimento e a difusão da inovação. A escala de análise, ilustrada na Figura 1 em um gráfico do tipo teia de aranha, é a do tipo Lickert, escalonada em 5 pontos. 
Figura 1 - Escala do questionário do Radar da Inovação

\section{Grau de Inovação}

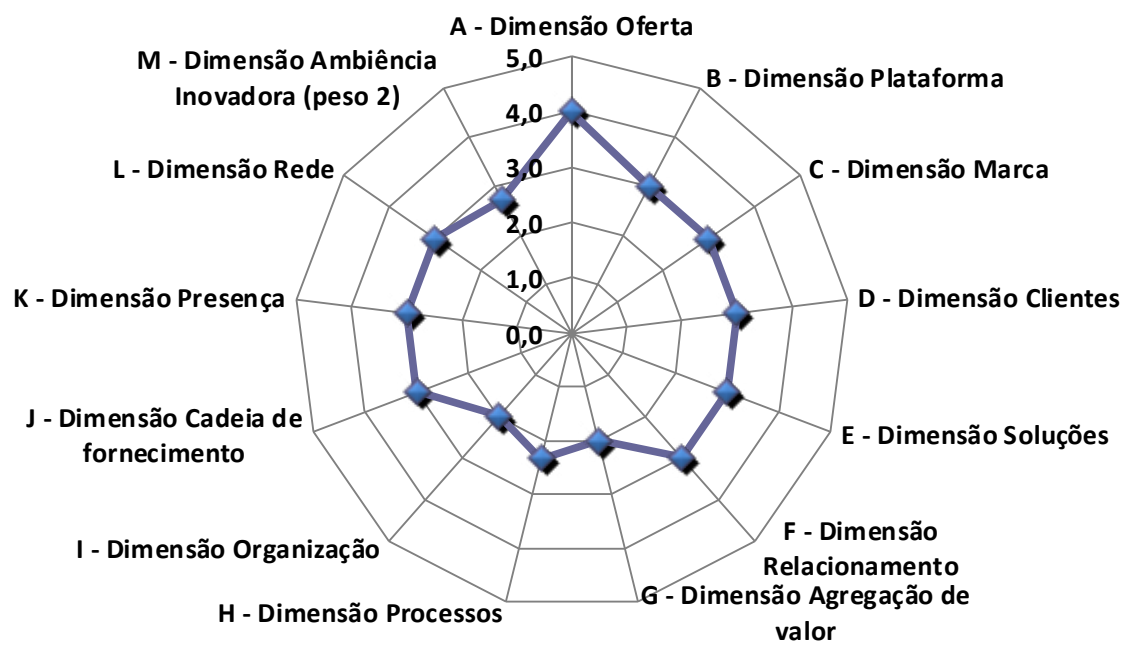

Fonte: Bachmann e Destefani (2008)

Essa escala de medida do grau de inovação (Figura 1) indica que a menor pontuação é 0,0 e a maior pontuação é 5,0, em cada uma das 13 dimensões. As empresas com a pontuação igual ou superior a 4,0 são definidas como inovadoras sistêmicas, pois praticam sistematicamente a gestão da inovação. As empresas com a pontuação igual a 3,0 são definidas como inovadoras ocasionais, pois são as empresas que inovaram nos últimos três anos, porém não têm sistematização do processo. As empresas com a pontuação igual ou superior a 1,0 e inferior a 3,0 são definidas como pouco ou nada inovadoras, já que são empresas que inovaram pouco, segundo essa métrica. E quando o escore é igual a 0,0 são as empresas que não inovam (SEBRAE, 2010).

A coleta de dados foi realizada in loco e iniciou em agosto de 2015 tendo sido encerrada em agosto de 2017. As respostas foram concedidas pelos proprietários das empresas por escrito no questionário e em seguida os dados coletados migraram para uma planilha em Excel, na qual foi construída a base de dados para análise. A análise apresentada em sequência em conjunto com a discussão dos resultados foi baseada em estatística descritiva.

\section{Resultados e Discussão}

A seguir apresenta-se a análise do estágio inicial (R0) do primeiro diagnóstico obtido pela aplicação da ferramenta Radar da Inovação nas empresas em estudo, conforme a Figura 2: 
Figura 2 - Grau da inovação

DIAGNÓSTICO RADAR DA INOVAÇÃO- R0

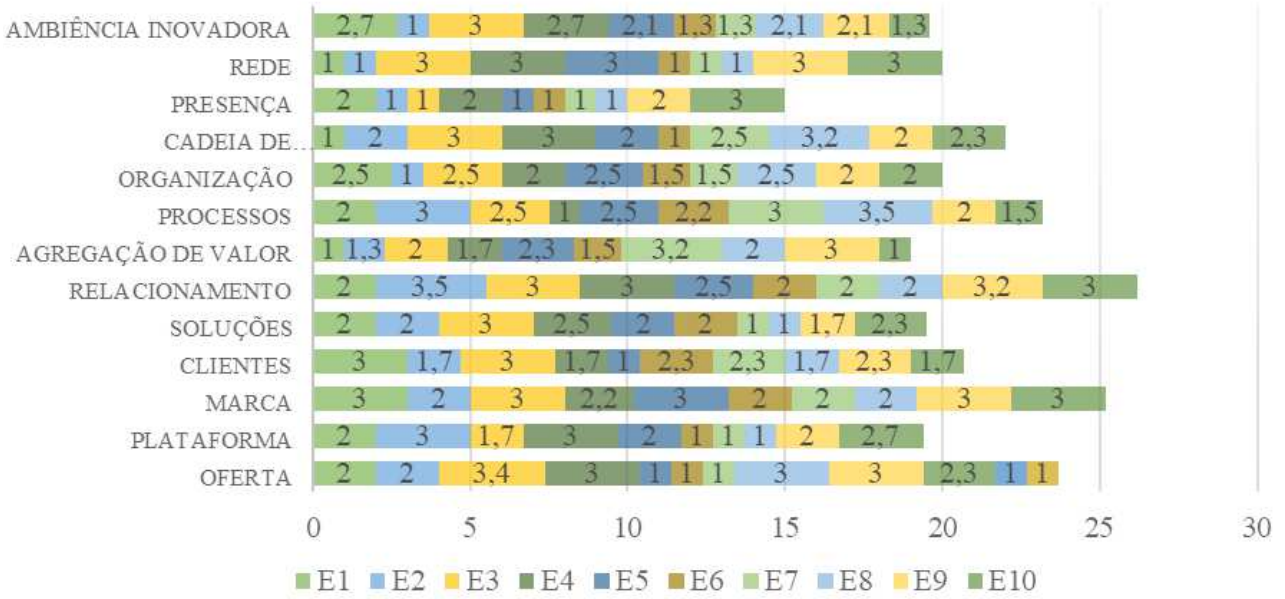

Fonte: Elaborada pelos autores deste artigo (2018)

De acordo com a escala apresentada na Figura 2, as empresas analisadas demonstraram serem ocasionalmente inovadoras. Assim, desenvolvem ações esporádicas para interagir no mercado, o que aponta para a possibilidade de grandes oportunidades de inovação. A situação inicial em que as empresas do setor de comércio varejista no segmento de vestuário se encontravam, deu-se em razão do pouco ou nenhum desenvolvimento de ações inovadoras. Porém, durante o atendimento essa primeira realidade começou a ser revertida, tendo em vista a relevância do assunto, para o crescimento do desempenho favorável dos empreendimentos.

Nesse sentido, como mostra a Figura 3, foram implantadas as ações de inovações realizadas nas empresas atendidas.

Figura 3 - Ações realizadas pelo Programa ALI

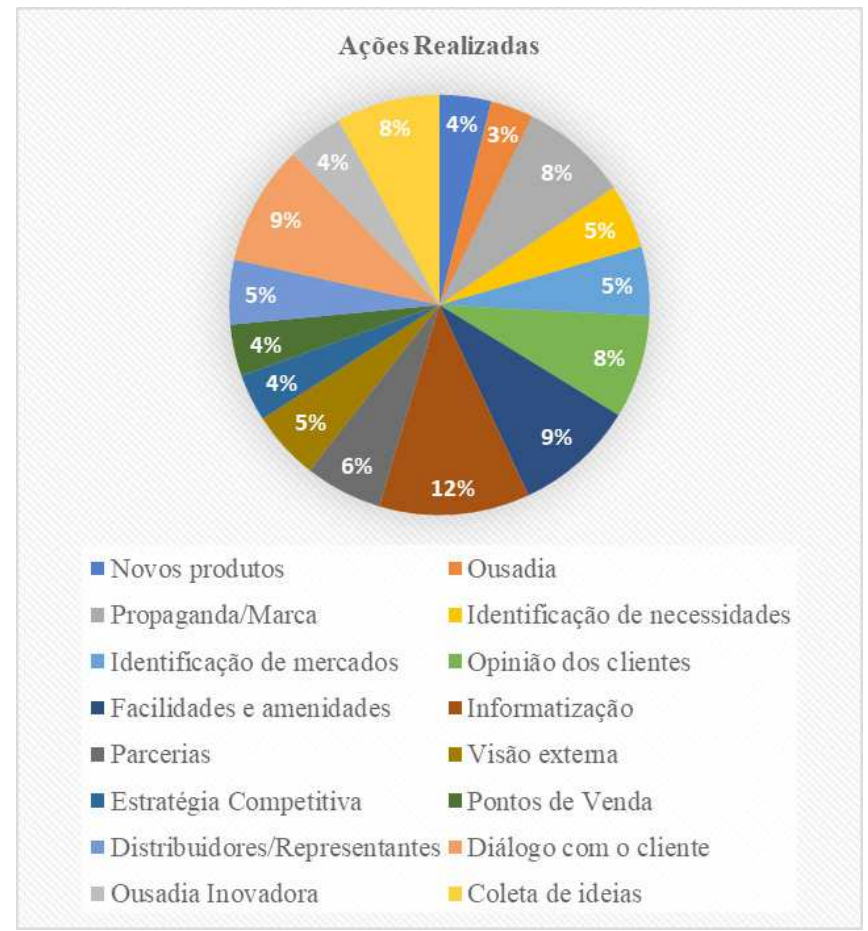

Fonte: Elaborada pelos autores deste artigo (2018) 
No estágio intermediário (R1) quando da aplicação do segundo diagnóstico e, depois da implantação de ações inovadoras na empresa por parte do acompanhamento do Programa ALI, as empresas já começaram a apresentar alguns resultados. Assim a Figura 4 ilustra a comparação e finalização do último diagnóstico (R2), aplicado nas empresas, depois da implantação de mais algumas ações em inovação.

Figura 4 - Grau geral de inovação - Radar da Inovação

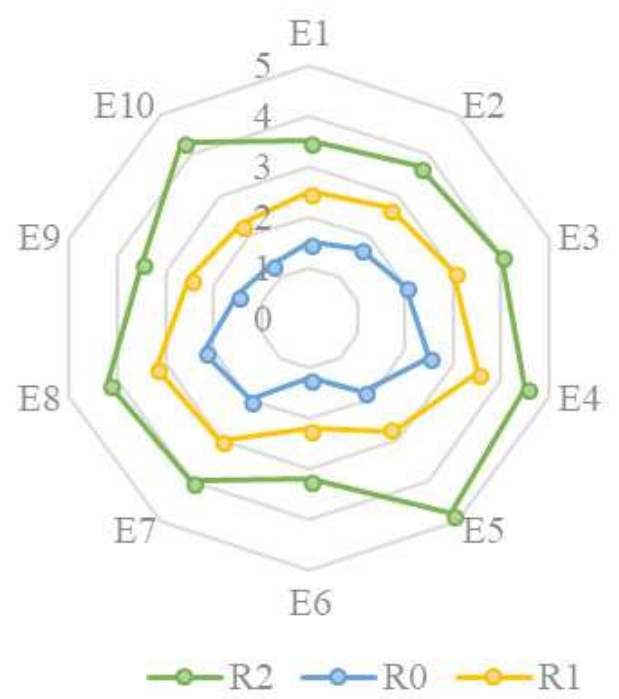

Fonte: Elaborada pelos autores deste artigo (2018)

A Figura 4 é referente à análise final do crescimento no Radar da Inovação durante o acompanhamento do Programa ALI. O que se pode perceber comparando principalmente à Figura 2 é que inicialmente, as empresas atendidas, começaram com pouco desenvolvimento em ações de inovação, as com a adesão ao Programa, sendo então aplicadas algumas ações, melhoraram sua situação no que se refere à apresentação de resultados positivos nas dimensões analisadas pela ferramenta.

\section{Considerações Finais}

Nesse trabalho, buscou-se desenvolver o tema inovação, alinhado a execução de uma política pública aplicada à realidade de MPEs do setor de vestuário, abordando os principais fatores envolvidos, as características, a avaliação de resultados e o estabelecimento da inovação. O Programa ALI do SEBRAE adota a utilização da ferramenta da análise Radar da Inovação, composta de dimensões que objetivam auferir se resultados foram gerados, a fim de analisar o grau de inovação existente ou não, nas empresas atendidas. Com isso o objetivo traçado pelo estudo foi o de analisar como o ciclo de 2015 a 2017 do Programa ALI está favorecendo o avanço de ações de inovação de 10 estabelecimentos de três cidades da Região Metropolitana de Recife. Os resultados encontrados indicam que esse objetivo foi alcançado, pois mostrou que as empresas analisadas estão saindo da posição de desenvolvedoras de inovação, por meio ocasional, e indo para a posição das que inovam sistematicamente.

O grande desafio observado, em relação à inovação presente nas MPEs, foi o de despertar a cultura da inovação nesses estabelecimentos, com o objetivo de que passem a coletar as 
informações para, posteriormente, serem desenvolvidas ações mais eficazes, capazes de transformar as informações obtidas em oportunidades de negócios. Essa conclusão é evidenciada, principalmente, pelo baixo empenho das empresas em concluírem o ciclo de avaliação proposto pela ferramenta Radar de Inovação. Porém, avalia-se, que mesmo que algumas MPEs não tenham concluído o atendimento, percebeu-se certa inquietação para adoção de inovações, o que pode ocasionar, posteriormente, que essas empresas venham a dar continuidade na aplicação de medidas inovadoras.

Os resultados também permitem inferir que o Programa ALI é uma ferramenta de política pública eficiente e eficaz dirigida ao desenvolvimento empresarial local, pois possibilita a implementação, pelos empresários, de ações e estratégias voltadas à criação, adoção e difusão de inovação, capazes de gerar emprego e renda, e assim mudar a realidade socioeconômica de uma região com base em ganhos econômicos positivos advindos de sua aplicação. Nesse sentido, uma provável contribuição deste estudo é que em futuras versões do Programa ALI, outros empresários possam vir a aderir ao acompanhamento diante dos resultados auferidos por esta pesquisa nessa ambiência inovadora.

Por fim, verifica-se que as empresas que deram continuidade e permaneceram no Programa ALI e concluíram o ciclo de três rodadas de aprimoramento, conseguiram aplicar medidas de inovação de maneira mais efetiva, conforme demonstradas nos resultados apresentados. Esse resultado também demonstra que apesar de algumas dificuldades em incorporar a inovação em produtos, serviços, processos e até mesmo na gestão, que algumas empresas possuíam inicialmente, houve determinação e criação de uma ambiência inovadora, para que os processos fossem aplicados e dessem resultados positivos ao final do ciclo.

Como viés aponta-se a necessidade de se incluir outros setores e/ou ampliar o tamanho da amostra. Nesse sentido, como sugestão de estudos futuros avalia-se que uma pesquisa possa incorporar essa ampliação do aspecto metodológico. Também se vislumbra comparar o Programa ALI, com algum programa internacional voltado para a mesma finalidade, a fim de se sopesar seus resultados frente a uma experiência internacional. Por fim, conclui-se que os resultados aqui obtidos não esgotam nem o tema nem a pesquisa no lócus de estudo escolhido e que o Programa ALI, enquanto política pública voltada à inovação, é bem sucedido e aplicado de maneira eficaz, com a sua implementação nas MPEs atendidas.

\section{Referências}

ANDREASSI, T. Gestão da inovação tecnológica. São Paulo: Thomson Learning, 2007. (Coleção Debates em Administração).

BACHMANN, D. L.; DESTEFANI, J. H. Metodologia para estimar o grau das inovações nas MPE. Curitiba: [s.n.], 2008.

BES, F. T.; KOTLER, P. A bíblia da inovação: princípios fundamentais para levar a cultura da inovação contínua às organizações. São Paulo: Lua de Papel, 2011.

CARVALHO, G. D. G. et al. Radar da inovação como ferramenta para o alcance de vantagem competitiva para micro e pequenas empresas. Revista de Administração e Inovação, São Paulo, v. 12 , n. 4 , p. $162-186,2015$. 
CARVALHO, M. M. Inovação: estratégias e comunidades de conhecimento. São Paulo: Atlas, 2009.

CAVALCANTI FILHO, A. M.; OLIVEIRA, M. R. G.; CAVALCANTI, A. M. Análise do desempenho em inovação das micro e pequenas empresas de TIC em Pernambuco. Revista Brasileira de

Administração Científica, Aquidabã, v. 3, n. 2, p. 41-56, 2012.

FARAH, M. F. S. Policy analysis at the municipal level of government. In: VAITSMAN, J.; RIBEIRO, J. M.; LOBATO, L. Policy analysis in Brazil. Bristol: University of Bristol, 2013.

FIGUEIREDO, P. N. Gestão da inovação: conceitos, métricas e experiências de empresas no Brasil. Rio de Janeiro: LTC, 2013.

HAIR, J. F. et al. Fundamentos de métodos de pesquisa em Administração. Porto Alegre: Bookman, 2005.

INSTITUTO BRASILEIRO DE GEOGRAFIA E ESTATÍSTICA (IBGE). Produto Interno Bruto. Brasília, DF: IBGE, 2017.

KON, A. Nova economia política dos serviços. São Paulo: CNPq, 2015.

INSTITUTO DE PESQUISA ECONÔMICA APLICADA(IPEA). ORGANIZAÇÃO PARA A COOPERAÇÃO E DESENVOLVIMENTO ECONÔMICO (OCDE). Condições para inovação no Brasil: uma revisão das principais questões e desafios políticos. Papel de suporte para o Workshop de Inovação para o Crescimento da Produtividade no Brasil. Brasília, DF: IPEA, 2015.

ORGANIZAÇÃO PARA COOPERAÇÃO E DESENVOLVIMENTO ECONÔMICO (OCDE). Manual de Oslo: diretrizes para a coleta e interpretação de dados sobre inovação. 3. ed. Rio de Janeiro: FINEP, 2007.

PAREDES, B. J. B.; SANTANA, G. A.; FELL, A. F. A. Um estudo de aplicação do radar da inovação: o grau de inovação organizacional em uma empresa de pequeno porte do setor metal-mecânico.

Navus-Revista de Gestão e Tecnologias, [S.1.], v. 4, n. 1, p. 76-88, 2014.

PORTER, M. E. Competição: estratégias competitivas essenciais. Rio de Janeiro: Campus, 1999.

RUA, M. G. As políticas públicas e a juventude dos anos 90. In: MINISTÉRIO DO PLANEJAMENTO E ORÇAMENTO. COMISSÃO NACIONAL DE POPULAÇÃO E DESENVOLVIMENTO. Jovens acontecendo na trilha das políticas públicas. vol. 2. Brasília, DF: CNPD, 1998. p. 731-752.

SAWHNEY, M.; WOLCOTT, R. C.; ARRONIZ, I. The 12 different ways for companies to innovate. MIT Sloan Management Review, [S.1.], v. 47, n. 3, p. 75-81, 2006.

SCHUMPETER, J. A. A teoria do desenvolvimento econômico. São Paulo: Nova Cultural, 1988.

SECCHI, L. Políticas públicas: conceitos, esquemas de análises, casos práticos. São Paulo:

Cengage Learning, 2012.

SERVIÇO BRASILEIRO DE APOIO ÀS MICRO E PEQUENAS EMPRESAS (SEBRAE). Gestão da inovação: inovar para competir. Brasília, DF: SEBRAE, 2010.

SERVIÇO BRASILEIRO DE APOIO ÀS MICRO E PEQUENAS EMPRESAS (SEBRAE); DEPARTAMENTO INTERSINDICAL DE ESTATÍSTICA E ESTUDOS SOCIOECONÔMICOS (DIEESE). Anuário do trabalho na micro e pequena empresa: 2013. 6. ed. Brasília, DF: DIEESE, 2013.

SOUZA, C. Políticas públicas: uma revisão da literatura. Sociologias, Porto Alegre, v. 8, n. 16, p. 20-45, 2006. 
WARWICK, K. Beyond industrial policy: emerging issues and new trends. OECD Science Technology and Industry Policy Papers, n. 2. Paris: OECD, 2013.

\section{Sobre os autores}

\section{Patricia da Silva Nogueira}

E-mail: patynogueiras@gmail.com

Mestranda em Propriedade Intelectual e Transferência de Tecnologia para Inovação (PROFNIT) na Universidade Federal de Pernambuco (UFPE); especialista lato sensu em Master of Business Administration (MBA) em Gestão Estratégica de Marketing, pela UFPE; e graduada em Comunicação Social/Jornalismo.

Endereço Profissional: Avenida Fagundes Varela, 445 - Jardim Atlântico, Olinda, PE. CEP: 53140-080.

\section{Sueli Menelau de Novais}

E-mail: suelimenelau@gmail.com

Doutora; mestre; e graduada em Administração.

Endereço profissional: Av. Prof. Moraes Rego, 1235 - Cidade Universitária, Recife, PE. CEP: 50670-901.

\section{Alessandra Sousa Cordeiro de Sá}

E-mail: alessandra_s.sa@hotmail.com

Mestranda do Programa de Pós-Graduação em Propriedade Intelectual e Transferência de Tecnologia para a Inovação (PROFNIT), na Universidade Federal de Pernambuco (UFPE); especialista em Processamento de Frutas e Hortaliças; e graduada em Tecnologia em Alimentos, ambos pelo Instituto Federal de Educação, Ciência e Tecnologia do Sertão Pernambucano.

Endereço profissional: Rua Antônio Gomes de Sá, 21 - Vila Eduardo, Petrolina, PE. CEP: 56328-625.

\section{Evelyn Siqueira Bezerra da Silva}

E-mail: evelynsbs05@gmail.com

Mestranda em Propriedade Intelectual e Transferência de Tecnologia para Inovação (PROFNIT), pela Universidade Federal de Pernambuco (UFPE).

Endereço profissional: Rua Alberto Barreto 74, Centro - Jaboatão do Guararapes, PE. CEP: 54410-222. 